\title{
Correction to: Malnutrition, assessed by the Global Leadership Initiative on Malnutrition (GLIM) criteria but not by the mini nutritional assessment (MNA), predicts the incidence of sarcopenia over a 5-year period in the SarcoPhAge cohort
}

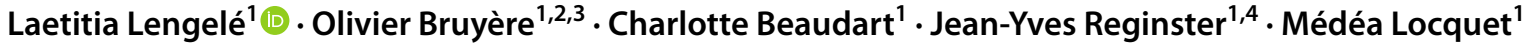

Accepted: 31 May 2021 / Published online: 13 July 2021

(c) Springer Nature Switzerland AG 2021

\section{Correction to: Aging Clinical and Experimental Research https://doi.org/10.1007/s40520-021-01880-5}

In the original publication of the article was wrongly published as.

"Malnutrition, assessed by the Global Leadership Initiative on Malnutrition (GLIM) criteria but not by the mini nutritional assessment (MNA), predicts the incidence of sarcopenia over a 5-year in the SarcoPhAge cohort"

The corrected title is.

"Malnutrition, assessed by the Global Leadership Initiative on Malnutrition (GLIM) criteria but not by the mini nutritional assessment (MNA), predicts the incidence of sarcopenia over a 5-year period in the SarcoPhAge cohort"

The original article has been updated.

Publisher's Note Springer Nature remains neutral with regard to jurisdictional claims in published maps and institutional affiliations.

The original article can be found online at https://doi.org/10.1007/ s40520-021-01880-5.

Laetitia Lengelé

llengele@uliege.be

1 WHO Collaborating Centre for Public Health Aspects of Musculoskeletal Health and Aging, Division of Public Health, Epidemiology and Health Economics, University of Liège, CHU—Sart Tilman, Quartier Hôpital, Avenue Hippocrate 13 (Bât. B23), 4000 Liège, Belgium

2 Department of Sport Rehabilitation Sciences, University of Liège, 4000 Liège, Belgium

3 Physical, Rehabilitation Medicine and Sports Traumatology, University Hospital of Liège, SportS2, 4000 Liège, Belgium

4 Biochemistry Department, College of Science, King Saud University, Riyadh 11451, Saudi Arabia 